\title{
Evidencias y salud: ¿hacia dónde va el sistema de salud en México?
}

\author{
Guillermo Soberón-Acevedo, PhD, (1) Cuauhtémoc Valdés-Olmedo, MSc. (2)
}

$\mathrm{L}$ a generación de evidencias para el diseño de políticas ha sido un factor crucial para el avance y mejora de los sistemas de salud en el mundo. Desde principios de los noventa han sido la brújula para identificar los ejes rectores de las reformas que han venido enfrentando los sistemas de salud en diversos países del orbe.

México es un claro ejemplo de ello y es por eso que la prestigiosa revista The Lancet dedicó espacio a siete artículos analíticos y a dos comentaristas internacionales para documentar su experiencia más reciente de reforma y decantar las lecciones aprendidas más replicables y pertinentes. Es preciso, en el marco de esta traducción al español de la misma serie y a la luz de un cambio de administración del Gobierno Federal en México, que tuvo lugar en diciembre de 2006, considerar los resultados documentados en The Lancet en su contexto histórico y en cuanto a las lecciones a futuro para el país.

Cabe mencionar un antecedente relevante para lo que se discute: en agosto de 1981 se creó la Coordinación de los Servicios de Salud de la Presidencia de la República que recibió la encomienda de "determinar los elementos técnicos, financieros, jurídicos y administrativos de los servicios públicos de salud a fin de integrarlos en un sistema nacional de salud que diera cobertura a toda la población". ${ }^{1}$

La propuesta de la Coordinación añadió cinco estrategias macro: la sectorización, la descentralización, la coordinación intersectorial de los servicios, la participación comunitaria, así como la modernización de la Secretaría de Salubridad y Asistencia, para que cum- pliera, a su vez, la función rectora del sistema que le confirió la Ley General de Salud, para lo cual cambió, en 1985, su denominación a Secretaría de Salud. También se definieron cinco estrategias micro: el estudio y racionalización del financiamiento de la salud, de la investigación en salud, de la formación de recursos humanos para la salud, de la información en salud, y de los insumos para la salud. Estas diez estrategias más la renovación legislativa fueron connotadas como "el cambio estructural de la salud". La renovación legislativa comprendió: la incorporación del derecho a la protección de la salud en nuestra Carta Magna, su ley reglamentaria, la Ley General de Salud, las Leyes Estatales de Salud, la definición de ocho reglamentos que sustituyeron a 80 farragozos ordenamientos y la emisión de normas técnicas. ${ }^{2}$

Por lo mismo, este comentario se dedica a reflexionar sobre los próximos pasos que habrán de darse para encaminarnos hacia un seguro universal en salud en México. Invitamos a los lectores y estudiantes de los sistemas de salud a hacer lo mismo referente a los múltiples caminos y lecciones que da esta serie titulada "la reforma del sistema de salud mexicano".

Con el informe de 1993 del Banco Mundial, ${ }^{3}$ diversos organismos tanto públicos como privados y entre ellos la Fundación Mexicana para la Salud (FUNSALUD), comenzaron un ejercicio de análisis y diagnósticos, basados en técnicas avanzadas como el peso de la enfermedad. En 1994, FUNSALUD fue líder a nivel mundial a aplicar, a nivel local, estas metodologías en el marco del proyecto "Economía y Salud", bajo la dirección de Julio Frenk, en el cual se incluyó una

(I) El Colegio Nacional; Comisión Nacional de Bioética; Fundación Mexicana para la Salud, México.

(2) Fundación Mexicana para la Salud, México. 
serie de recomendaciones sobre políticas para el mejoramiento del sistema de salud. ${ }^{4}$

El trabajo Economía y Salud dio como uno de sus resultados principales el análisis del financiamiento a la salud y en él se puso de manifiesto la importancia antes no percibida- que, en el sistema de salud mexicano, tiene el gasto privado en el financiamiento a la salud.

En el año 2000, del Informe sobre la Salud en el Mundo "Mejorar el Desempeño de los Sistemas de Salud" de la Organización Mundial de la Salud (OMS) México ocupó la posición $61 .{ }^{5} \mathrm{El}$ ordenamiento mundial que se hizo sobre los indicadores base del marco de la OMS evidenció, para el caso de México, que el diagnóstico hecho desde 1994 en México había identificado el punto más débil del sistema de salud -el alto nivel de financiamiento a la salud basado en el gasto privado, principalmente del bolsillo de los hogares. Se evidenció un alto grado de inequidad e insuficiencia de los recursos.

Durante el sexenio 2000-2006 bajo el liderazgo del Secretario de Salud Julio Frenk, el tratamiento para remediar la enfermedad de la falta de protección financiera y la fragmentación del sistema entre asegurados y no-asegurados, fue una reforma estructural del sistema de salud en México. Esta reforma dio como frutos la creación del Sistema de Protección Social en Salud que tomaba como principal instrumento de operación al Seguro Popular de Salud. Por su parte, la iniciativa de reforma tomó en cuenta una cadena acumulativa de evidencias señaladas en el Informe Economía y Salud y otros documentos de FUNSALUD, en el informe de la OMS de 2000 y por un conjunto de investigaciones llevadas a cabo por la nueva administración en salud e investigadores de instituciones como el Instituto Nacional de Salud Pública y FUNSALUD.

Es esta una experiencia exitosa de aplicación de evidencia a la formulación de políticas públicas en la reforma que constituye el hilo conductor para los documentos que conforman la Serie de The Lancet y ahora de esta edición especial de Salud Pública de México y que dio lugar al Seminario "Evidencias para la Reforma de los Sistemas de Salud: Lecciones Globales a partir de Experiencias Nacionales" que tuvo lugar en la Ciudad de México del 4 al 6 de octubre de 2006.

La experiencia de México comprueba que las evidencias pueden servir como catalizadores para generar las reformas de los sistemas de salud. Por lo mismo, es menester retomar, a la luz de la situación actual de la reforma en México, los hallazgos de otro estudio. Hacemos referencia a una propuesta de reforma titulada "Hacia un México más saludable" que salió del trabajo de FUNSALUD entre 1998 y 1999 en seguimiento a la línea de análisis sobre economía y salud. ${ }^{6}$

A través del análisis resumido en el documento publicado por FUNSALUD en 2000, se dictaminó que mientras se mantuviesen integradas las funciones de financiamiento y prestación de los servicios en una misma institución, ésta seguiría actuando como juez y parte de un proceso que debe de ser supervisado y evaluado a fin de introducir correctivos e innovaciones que le permiten seguir mejorando. Dicho de otro modo, la separación del financiamiento y la prestación será un factor importante para incrementar la calidad de los servicios, atenuar la inequidad y aumentar la eficiencia.

Por otro lado, para asegurar un sistema nacional de salud equitativo, de calidad y eficiente, la reforma debería apoyarse en la creación de un seguro nacional de salud de carácter universal, en el cual no se discrimine entre poblaciones y de tipo solidario, en donde los recursos acompañen a las necesidades. La solidaridad, bien entendida, significa que, en el cuidado de la salud, los sanos paguen por los enfermos, los jóvenes por los de edad avanzada y los ricos por los pobres. Esto podrá lograrse con la fusión de la asignación de recursos financieros entre instituciones y la separación del financiamiento de la prestación.

Finalmente, la propuesta señala la importancia de fomentar la prestación plural -pública y privada- de los servicios de salud. Esto es un insumo importante para la creación de un seguro nacional de salud. La prestación plural constituye una poderosa herramienta la cual, junto con la separación de la prestación del financiamiento, dará un empujón decisivo a la eficiencia y la competitividad del sector salud pero también a la equidad y calidad de la prestación de los servicios. Muchas de las bases necesarias para dar este paso empezaron en el contexto de la reforma -en particular el fortalecimiento del sector público y la ampliación del proceso de certificación para asegurar la calidad de los servicios.

En resumen la propuesta "Hacia un México más saludable" da particular importancia a tres puntos clave: 1) la necesidad de la separación entre financiamiento a la salud y la prestación de los servicios; 2) la creación de un seguro universal de salud financiado a través de un fondo nacional de salud; y 3 ) fomentar la prestación plural de los servicios. Son estos los próximos pasos que tiene que tomar el sistema de salud en México, ya con la sólida base financiera que legó a México la reforma del 2003 y a la luz del compromiso actual de llevar esta reforma hacia la protección financiera universal. 


\section{Referencias}

I. Comisión de Salud y Seguridad Social, Coordinación de los Servicios de Salud y Universidad Nacional Autónoma de México. Hacia un Sistema Nacional de Salud. México, D.F., 1983.

2. Soberón G. El cambio estructural en la salud. Salud Publica Mex 1987;29:127-140.

3. Banco Mundial. Informe sobre el desarrollo mundial, 1993. Invertir en salud. Washington, D.C.: Banco Mundial, 1993.

4. Frenk J, Lozano R, González M. Economía y salud: propuesta para el avance del sistema de salud en México. Informe final. México, D.F::

Fundación Mexicana para la Salud, 1994.

5.World Health Organization. The World Health Report 2000. Health systems: Improving performance. Geneva:WHO, 2000.

6. Fundación Mexicana para la Salud. Hacia un México más saludable: una visión del sector privado, México, DF: FUNSALUD, 2000. 